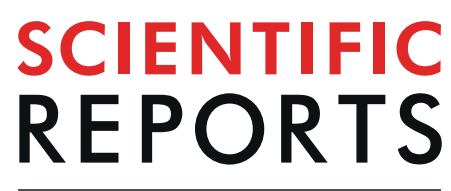

natureresearch

\title{
OPEN Characterization of biklflklf17- deficient zebrafish in posterior lateral line neuromast and hatching gland development
}

Received: 29 November 2018

Accepted: 29 August 2019

Published online: 26 September 2019

\begin{abstract}
Hiroaki Suzuki ${ }^{1}$, Tomoe Ishizaka ${ }^{1}$, Kanoko Yanagi ${ }^{1}$, Ryota Sone ${ }^{1}$, Yuto Sunaga ${ }^{2}$, Rie Ohga ${ }^{1}$ \&
\end{abstract} Atsuo Kawahara ${ }^{1}$

Krüpple-like factors (KIfs) are highly conserved zinc-finger transcription factors that regulate various developmental processes, such as haematopoiesis and cardiovascular development. In zebrafish, transient knockdown analysis of biklf/klf17 using antisense morpholino suggests the involvement of biklflklf17 in primitive erythropoiesis and hatching gland development; however, the continuous physiological importance of $k$ lf 17 remains uncharacterized under the genetic ablation of the $k l f 17$ gene among vertebrates. We established the $k$ lf17-disrupted zebrafish lines using the CRISPR/Cas 9 technology and performed phenotypic analysis throughout early embryogenesis. We found that the klf17-deficient embryos exhibited abnormal lateral line neuromast deposition, whereas the production of primitive erythrocytes and haemoglobin production were observed in the klf17-deficient embryos. The expression of lateral line neuromast genes, $k l f 17$ and s100t, in the klf17-deficient embryos was detected in posterior lateral line neuromasts abnormally positioned at short intervals. Furthermore, the klf17-deficient embryos failed to hatch and died without hatching around 15 days post-fertilization (dpf), whereas the dechorionated klf17-deficient embryos and wild-type embryos were alive at 15 dpf. The $k$ lf17-deficient embryos abolished hatching gland cells and Ctsl1b protein expression, and eliminated the expression of polster and hatching gland marker genes, he1.1, ctsl1b and cd63. Thus, the klf17 gene plays important roles in posterior lateral line neuromast and hatching gland development.

Krüpple-like transcription factors (Klfs), which are characterized by the Cys2-His2 zinc-finger motif at the C-terminus, are involved in various developmental processes, such as haematopoiesis and cardiovascular development $^{1,2}$. Phylogenetic analysis suggests that the Klf17 family belongs to a distinct branch closely related to Klf2 and Klf4 families, and mammalian Klf17 proteins remarkably diverged from those of other species ${ }^{3}$, showing rapid evolution in mammals. In mice, the Klf17 gene was identified as a germ cell-specific gene ${ }^{4}$. Recent accumulating evidence suggests that the Klf17 gene in mammals plays important roles in tumourigenesis ${ }^{5}$. The Klf17 gene is down-regulated in various human cancers $^{6}$, presumably leading to the epithelial-mesenchymal transition (EMT) and metastases. Importantly, the physiological function of Klf17 during mammalian embryogenesis is not fully understood.

klf17/biklf (blood island-enriched Krüpple-like factor) was originally identified as one of the zygotic-activated genes during zebrafish embryogenesis ${ }^{7}$. Zebrafish $k l f 17$ is expressed in the involuting axial mesoderm and polster during gastrulation and is subsequently expressed in the blood island/intermediate cell mass (ICM), hatching gland and lateral lines during organogenesis. Xenopus klf17/neptune is expressed in the hatching gland, cement gland and ventral blood island during embryogenesis ${ }^{8,9}$, whereas the chick Klf17 gene is expressed in the blood island ${ }^{10}$. Knockdown of zebrafish $k l f 17$ gene results in the impairment of primitive erythropoiesis and of hatching gland development ${ }^{11-13}$. The Xenopus klf17/neptune morphants exhibit loss of the hatching gland and otic vesicle and malformation of neural crest-derived cranial cartilage ${ }^{14}$.

${ }^{1}$ Laboratory for Developmental Biology, Center for Medical Education and Sciences, Graduate School of Medical Science, University of Yamanashi, Shimokato 1110, Chuo, Yamanashi, 409-3898, Japan. ${ }^{2}$ Department of Pediatrics, Faculty of Medicine, University of Yamanashi, Shimokato 1110, Chuo, Yamanashi, 409-3898, Japan. Hiroaki Suzuki and Tomoe Ishizaka contributed equally. Correspondence and requests for materials should be addressed to A.K. (email: akawahara@yamanashi.ac.jp) 

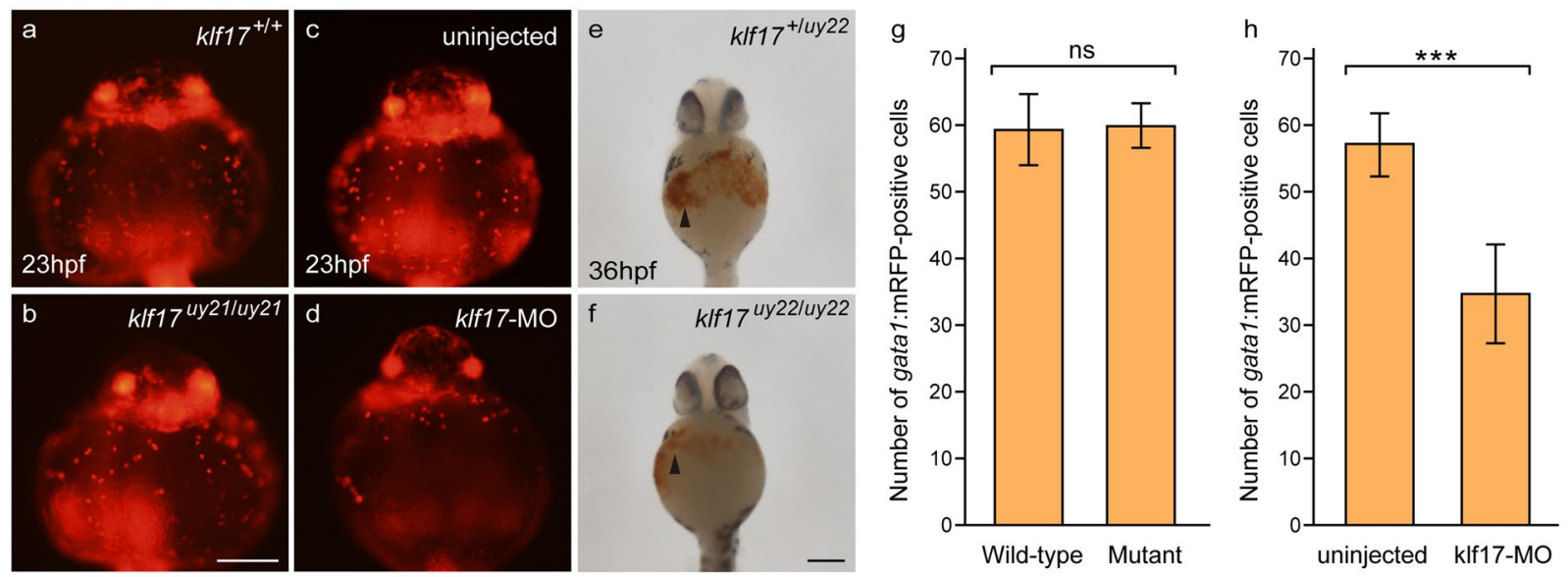

Figure 1. Primitive erythrocytes were observed in the klf17-deficient embryos. (a-d) Production of primitive erythrocytes in the klf17-deficient embryo and in the klf17-morphant embryo at $23 \mathrm{hpf}$. The number of erythrocytes visualized by the gata1:mRFP transgene was comparable in wild-type embryo $\left(k l f 17^{+/+}\right)(\mathbf{a})$ and the klf17-deficient embryo $\left(k l f 17^{u y 21 / u y 21}\right)$ (b) (ventral view, anterior up), whereas the number of erythrocytes was less in the klf17-morphant embryo (d). Scale bar, $200 \mu \mathrm{m}$. (e,f) Haemoglobin production in the klf17deficient embryos at $36 \mathrm{hpf}$. Haemoglobin production visualized by $o$-dianisidine staining (arrowheads) was detected in the wild-type embryo containing an intact allele $\left(k l f 17^{+/ u y 22}\right)$ embryo and in the $k l f 17$-deficient embryo (klf17 $7^{u 22 / u y 22}$ ) (ventral view, anterior up). After taking pictures, genotyping of individual embryos was performed by genomic PCR. Scale bar, $200 \mu \mathrm{m}$. (g) The number of gata1:mRFP-positive cells on the yolk in wild-type $(\mathrm{n}=19)$ and in the klf17-deficient embryos (mutant) $(\mathrm{n}=8)$ were counted. Error bars indicate standard deviation. ns, not significant. (h) The number of gata1:mRFP-positive cells on the yolk in uninjected $(\mathrm{n}=14)$ and in the klf17-molpholino $(10 \mathrm{ng})$-injected embryos $(\mathrm{n}=19)$ were counted. Asterisk indicates statistical significance. $* * * P<0.001$. Error bars indicate standard deviation.

Recent studies demonstrate that genetically gene-disrupted mutants and morphants induced by transient morpholino injection often exhibit distinct morphological phenotypes ${ }^{15}$. In fact, morpholino injection in zebrafish embryos often causes ectopic p53 induction ${ }^{16}$. Furthermore, undesirable off-target effects mediated by morpholino injection have been reported ${ }^{17}$. Transient knockdown analysis using morpholino injection is unsuitable for continuous in vivo analysis; therefore, we have generated the klf17-disrupted zebrafish mutants using the CRISPR/Cas9 technology and examined the continuous physiological function of $k l f 17$ throughout early embryogenesis.

\section{Results}

Primitive haematopoiesis in the $\boldsymbol{k l f 1 7 - d e f i c i e n t ~ e m b r y o s . ~ T o ~ e x a m i n e ~ t h e ~ p h y s i o l o g i c a l ~ f u n c t i o n ~ o f ~}$ $k l f 17$ during zebrafish early embryogenesis, we established the klf17-disrupted zebrafish lines using the genome editing technology, CRISPR/Cas9. Three klf17 alleles uy21, uy22 and uy23 with totally 20 base pairs (bp), $20 \mathrm{bp}$ deletions and a 28 bp insertion, respectively, were isolated (Supplemental Figs S1 and S2). Because the predicted Klf17 mutant proteins derived from mutant alleles lacked most of the coding domains including zinc fingers (Supplemental Fig. S3), they would be functionally disrupted.

Knockdown analysis of the klf17 gene using antisense morpholino suggests that the zebrafish $k l f 17$ gene is involved in primitive erythropoiesis development ${ }^{11-13}$. Therefore, we first examined the production of primitive erythrocytes monitored by gata 1:mRFP transgene and haemoglobin production by $o$-dianisidine staining in the klf17-deficient embryos. The number of erythrocytes on the yolk seemed to be comparable in wild-type $(\mathrm{n}=19)$ and $k l f 17$-deficient embryos $(\mathrm{n}=8)$ at 23 hours post-fertilization $(\mathrm{hpf})$, whereas primitive erythrocytes were decreased in the klf17-morphant $(\mathrm{n}=19)$ (Fig. 1). Production of haemoglobin and circulating blood cells were detected in the klf17-deficient embryos $(\mathrm{n}=10)$ and in wild-type embryos containing intact alleles $(\mathrm{n}=8)$ at $36 \mathrm{hpf}$ (Figs 1, S4 and Supplemental Movies 1-3). Furthermore, the expression of erythrocyte genes, gata1, $\beta_{e 3}$ globin (an embryonic globin) at 18-somite and $25 \mathrm{hpf}$ was comparable in wild-type and klf17-deficient embryos (Supplemental Fig. S5). The expression of myeloid cell marker lysozyme C (lyz) was comparably expressed in wild-type and the $k l f 17$-deficient embryos at $25 \mathrm{hpf}$. Thus, we did not observe the severe impairment of primitive erythropoiesis reported in the analysis of the klf17-morphant ${ }^{11,13}$.

Lateral line neuromast development in the $k l f 17-d e f i c i e n t$ embryos. Because $k l f 17$ gene is bilaterally expressed in the lateral line neuromasts ${ }^{18}$, we examined the differentiation of lateral line neuromasts in the klf17-deficient embryos at $54 \mathrm{hpf}$ using the fluorescent reagent 4-Di-2-ASP (Di-ASP) that can easily label differentiated neuromasts ${ }^{19}$. The posterior lateral line (PLL) primordium migrated caudally and periodically deposited neuromasts at regular five or six intervals and two or three terminal neuromasts (term) along the horizontal myoseptum of wild-type embryos. Deposition of the first PLL neuromast in the klf17-deficient embryos was posteriorly delayed (Fig. 2). The number of PLL neuromasts in the klf17-deficient embryos $(n=9)$ was less compared to 


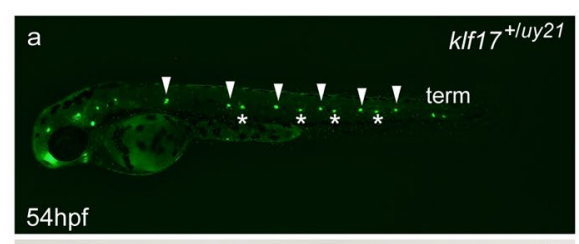

e
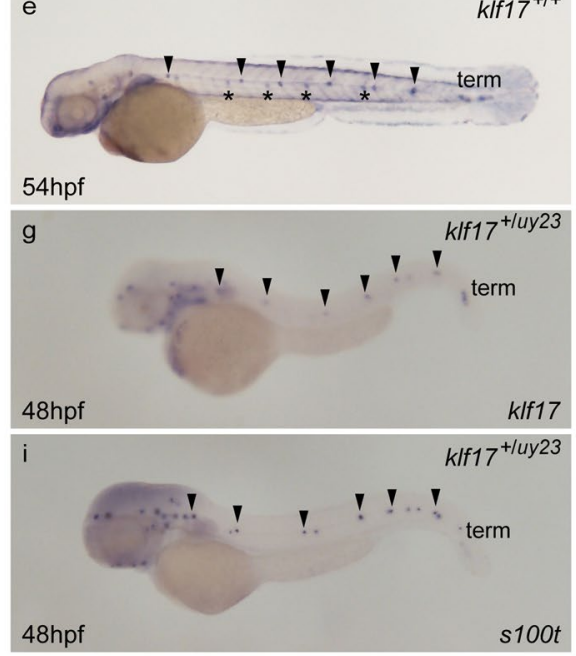
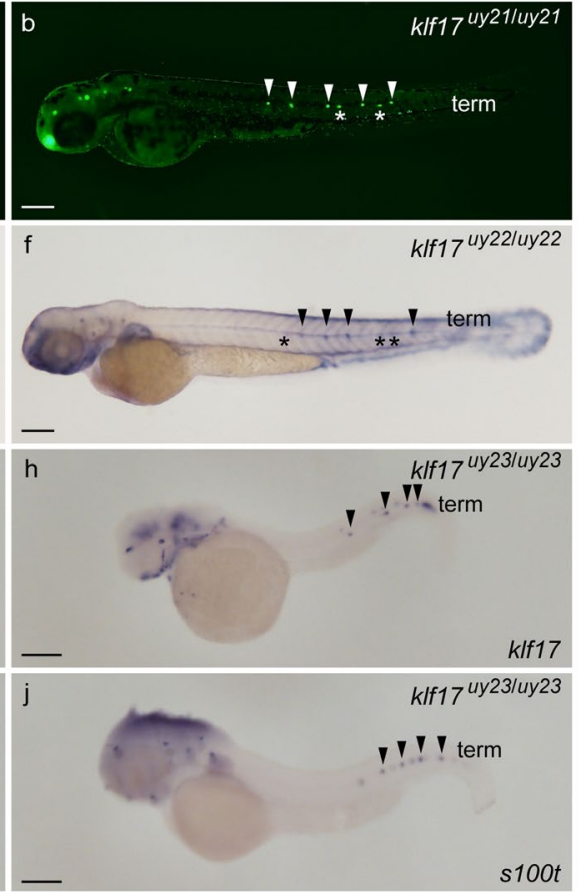
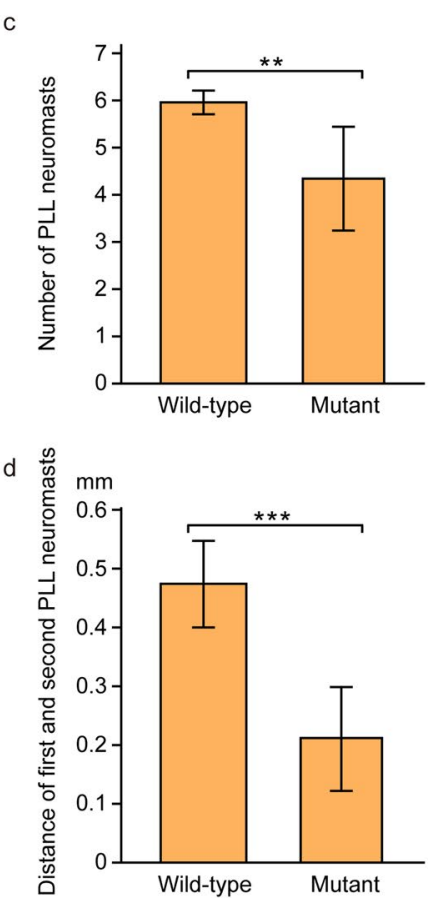

Figure 2. Abnormal PLL neuromast deposition in the klf17-deficient embryos. (a-d) Lateral line neuromasts stained with 4-Di-2-ASP (Di-ASP). PLL neuromasts were bilaterally stained with Di-ASP. White arrowheads indicate the position of PLL neuromasts on the left side. White asterisks indicate the position of PLL neuromasts on the right side. Term indicates the position of terminal neuromasts. The number of PLL neuromasts (c) and the distance between first and second neuromasts $(\mathbf{d})$ were measured in wild-type $(\mathrm{n}=16)$ and the $k l f 17$-deficient embryos $(\mathrm{n}=9)$. Asterisk indicates statistical significance between wild-type and the klf17deficient embryos. $* * P<0.01$. $* * * P<0.001$. Error bars indicate standard deviation. (e,f) Alkaline phosphatase accumulation in the lateral line neuromasts. Arrowheads indicate the position of PLL neuromasts on the left side. Asterisks indicate the position of PLL neuromasts on the right side. (g-j) The expression of lateral line genes, $k l f 17(\mathbf{g}, \mathbf{h})$ and $\operatorname{si00t}(\mathbf{i}, \mathbf{j})$, was examined by whole-mount in situ hybridization (WISH) at $48 \mathrm{hpf}$. (g,i) Wild-type embryos $\left(k l f 17^{+/ u y 23}\right)$. (h,j) klf17-deficient embryos $\left(k l f 17^{\text {uy23/uy23 }}\right)$. Arrowheads indicate the position of PLL neuromasts on the left side. All pictures showed lateral view, anterior left. After taking pictures, genotyping of individual embryos was performed by genomic PCR. Scale bar, $200 \mu \mathrm{m}$.

that of wild-type $(\mathrm{n}=16)$. The distance between first and second PLL neuromasts was short in the $k l f 17$-deficient embryos. Using visualization of alkaline phosphatase accumulation, the delay of the PLL neuromasts deposition was observed in the klf17-deficient embryos $(\mathrm{n}=5)$ compared to wild-type embryos $(\mathrm{n}=15)$ (Fig. 2e,f). Injection of klf17 mRNA (20 pg) in wild-type and the klf17-deficient embryos caused to axis defects at $36 \mathrm{hpf}$ (Supplemental Fig. S6). The expression of lateral line neuromast genes, $k l f 17$ and $s 100 t$, in the PLL neuromasts was located posteriorly in the klf17-deficient embryos (Fig. $2 \mathrm{~g}-\mathrm{j}$ ). Thus, the $k l f 17$ gene is required for proper PLL neuromasts deposition.

Hatching gland development in the klf17-deficient embryos. The klf17 gene is detected in the polster during gastrulation stages and in the hatching gland during organogenesis stages ${ }^{7}$. We found that the klf17-deficient embryos $(\mathrm{n}=19)$ failed to hatch at 3 days post-fertilization $(\mathrm{dpf})$ and $6 \mathrm{dpf}$, whereas wild-type embryos $(\mathrm{n}=17)$ hatched until $3 \mathrm{dpf}$ (Figs 3 and S4). Such hatching defects were obvious and are also observed in the klf17-morphant ${ }^{12}$.

The transient klf17-morphant is unsuitable for continuous in vivo analysis. We manually removed the chorion membranes from the klf17-deficient embryos and grew up them. We found that the klf17-deficient embryos $(n=20)$ died without hatching approximately $15 \mathrm{dpf}($ Fig. 3$)$. Wild-type embryos $(\mathrm{n}=12)$ and the dechorionated-klf17-deficient embryos $(\mathrm{n}=19)$ and were alive. Therefore, we examined the function of $k l f 17$ in hatching gland development.

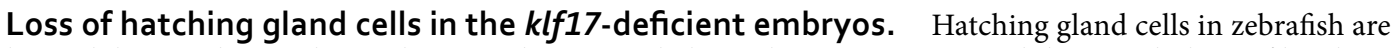
located deep to the enveloping layer on the pericardial membrane. We examined the morphology of hatching gland cells using cross sections of wild-type and the klf17-deficient embryos at $48 \mathrm{hpf}$. Hatching gland cells visualized by haematoxylin and eosin $(\mathrm{HE})$ staining were observed in wild-type embryos $(n=9)$, whereas the klf17-deficient embryos $(n=10)$ were completely missing the hatching gland cells (Fig. 4). Next, we examined the protein expression of Cathepsin L $1 \mathrm{~b}$ (Ctsl1b) that is one of hatching enzymes. Using anti-Ctsl1b immunohistochemistry, Ctsl1b protein was predominantly expressed in the hatching gland cells of wild-type embryo (Fig. 5). In clear contrast to the wild-type, Ctsl1b expression was not detected in the klf17-deficient embryos. 


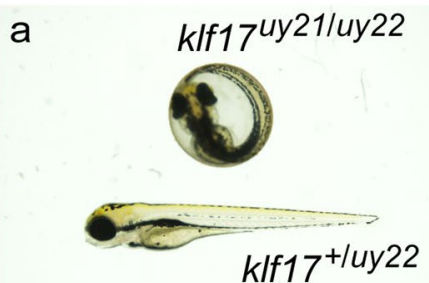

b

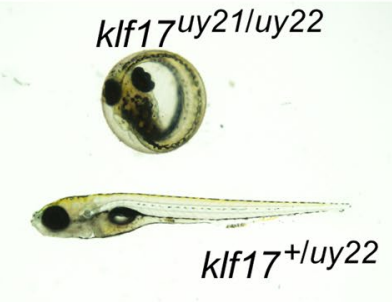

e

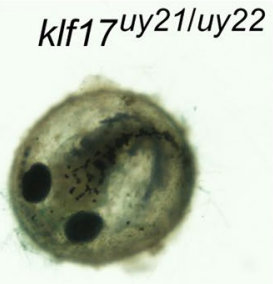

$3 \mathrm{dpf}$

C
$6 \mathrm{dpf}$

$k l f 17^{+/+} \quad \mathrm{d}$ d
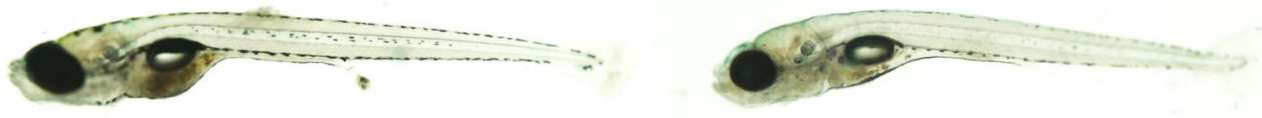

\section{$15 \mathrm{dpf}$}

Figure 3. klf17-deficient embryos failed to hatch during zebrafish embryogenesis. (a,b) Hatching-deficient phenotype in the klf17-deficient embryos at $3 \mathrm{dpf}(\mathbf{a})$ and $6 \mathrm{dpf}(\mathbf{b})$. The klf17-deficient embryo $\left(k l f 17^{\text {uy21/uy22}}\right)$ failed to hatch at 3 and $6 \mathrm{dpf}$, whereas wild-type $\left(k l f 17^{+/ u y 22}\right)$ embryo hatched until $3 \mathrm{dpf}$. Scale bar, $1 \mathrm{~mm}$. (c-e) Dechorionated-klf17-deficient embryos were alive at $15 \mathrm{dpf}$. Wild-type embryo $\left(k l f 17^{+/+}\right)(\mathbf{c})$ and the dechorionated- $k l f 17$-deficient embryo $\left(k l f 17^{\text {uy21/uy22 }}\right)(\mathbf{d})$ were both alive at $15 \mathrm{dpf}$, whereas the $k l f 17$-deficient embryo $\left(k l f 17^{\text {uy21/uy22}}\right.$ ) (e) died without hatching. Scale bar, $2 \mathrm{~mm}$. After taking pictures, genotyping of individual embryos was performed by genomic PCR.

Next, we examined the expression of polster and hatching gland genes. Consistent with the morphological hatching gland defects, the expression of he1.1 (hatching enzyme 1), ctsl1b, cd63 and klf17 in the klf17-deficient embryos was reduced in the polster of the bud stage embryos (Fig. 6), and was not detected in the hatching gland at $25 \mathrm{hpf}$ (Fig. 7). We examined morphology of polster at bud stage. We found that the polster was not dected in the klf17-deficient embryo (Supplemental Fig. S7). These results suggested that the klf17 gene plays important roles in the polster and hatching gland development in zebrafish.

\section{Discussion}

Transient knockdown analysis of klf17 suggests the involvement of klf17 in primitive erythropoiesis and hatching gland development in zebrafish ${ }^{11-13}$. In this study, we have generated the klf17-deficient zebrafish lines and examined the physiological function of klf17 during early embryogenesis. Although the klf17-morphant exhibits severe defects in primitive erythropoiesis ${ }^{11,13}$, both primitive erythrocytes and haemoglobin production in the klf17-deficient embryos were observed (Figs 1, S4 and Supplemental Movies 1-3). Reasons of these discrepancies of haematopoietic defect between the klf17-morphants and klf17-deficient embryos are not clear at present. Recent studies found that $k l f 1, k l f 2 a, k l f 3, k l f 6 a$ and $k l f 8$ in addition to klf17 are expressed in the ICM ${ }^{20}$. Knockdown of $k l f 3$ or klf6a induced a blockage of erythrocyte maturation. One possible explanation is that other $k l f$ genes expressed in the ICM function redundantly in primitive erythropoiesis in zebrafish. Multiple $k l f$ genes disruption based on the klf17-deficient zebrafish lines will be required to clarify the possibility.

Although $k l f 17$ is known to be bilaterally expressed in lateral line neuromasts ${ }^{18}$, the function of $k l f 17$ in lateral line neuromasts is not fully understood. PLL primordium migrates along the horizontal myoseptum and periodically deposits 5 or 6 neuromasts and finally forms 2 or 3 terminal neuromasts at the tip of the tail ${ }^{21}$. Di-ASP staining analysis revealed that the deposition of first PLL neuromast was delayed posteriorly (Fig. 2). The number of PLL neuromasts was less and the distance between first and second PLL neuromasts was short in the klf17-deficient embryos. The delayed deposition of PLL neuromasts was also confirmed by visualization of alkaline phosphatase accumulation in differentiated PLL neuromasts. Furthermore, the expression of $k l f 17$ and $s 100 t$ at $48 \mathrm{hpf}$ was detected in PLL neuromasts abnormally positioned at short intervals. Because the other $k l f$ genes except for klf17 are not detected in lateral line neuromasts, the induction of mutated form of $k l f 17$ mRNA in the klf17-deficient embryos may affect the deposition and differentiation of PLL neuromasts. Although the molecular mechanism of PLL neuromast differentiation remains unclear, this study demonstrates that the klf17 gene is required for proper PLL neuromast deposition.

The klf17 is predominantly expressed in the hatching gland in zebrafish and Xenopus ${ }^{7-9}$. We found that the klf17-deficient embryos failed to hatch during embryogenesis (Figs 3 and S4). Such a hatching defect is consistent with that of zebrafish klf17-morphants and Xenopus klf17-morphants ${ }^{13,14}$. Our continuous observation revealed that the $k l f 17$-deficient embryos died without hatching approximately $15 \mathrm{dpf}$. Notably, the dechorionated-klf17-deficient embryos were alive at $15 \mathrm{dpf}$ (Fig. 3). The zebrafish $k l f 3$ gene is weakly expressed compared with the expression of klf17 in the hatching gland, but the other klf genes except for klf 3 and $k l f 17$ are not detected in the hatching gland ${ }^{20}$; therefore, the klf 17 would play an essential role in the hatching gland 


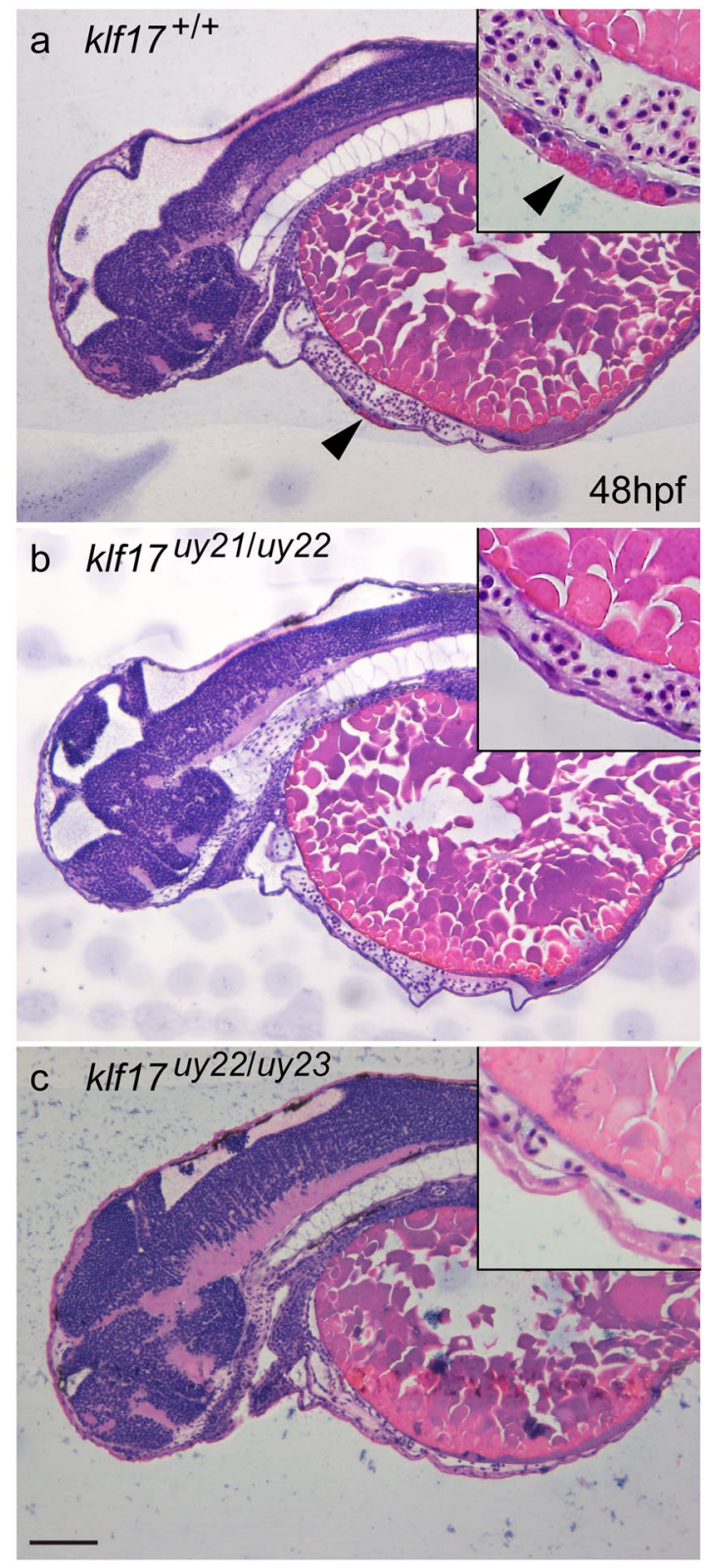

Figure 4. Loss of hatching gland cells in the klf17-deficient embryos. (a-c) Cross sections of haematoxylin and eosin (HE)-stained embryos at 48 hpf: wild-type $\left(k l f 17^{+/+}\right)$(a) klf17-deficient embryos: $k l f 17^{\text {uy21/uy22 }}$ (b) and $k l f 17^{\text {uy22/uy23 }}$. (c) Hatching gland cells observed in wild-type embryo were missing in the klf17-deficient embryos. Arrowheads indicate the position of hatching gland cells. Genomic DNA was isolated from individual caudal fins, with genotyping was performed by genomic PCR. Scale bar, $100 \mu \mathrm{m}$.

development. Using mammalian cell lines, Klf17 was recently identified as a negative regulator of metastasis in breast cancer ${ }^{6}$. Klf17 is predominantly expressed in testis and ovary in mice ${ }^{4}$; however, the physiological function of mammalian Klf17 is not fully understood. Because the hatching gland does not exist in mammals, further loss-of-function analysis using Klf17-disrupted mice will be required to understand the developmental function of mammalian Klf17.

Cross section analysis of HE-stained zebrafish wild-type embryos identified the presence of hatching gland cells with cytoplasmic granules ${ }^{22}$, presumably containing hatching enzymes that digest the chorion membrane. In clear contrast to that analysis, the hatching gland cells were not observed in the klf17-deficient embryos (Fig. 4). Anti-Ctsl1b immunohistochemistry revealed that the Ctsl1b protein, which is one of hatching enzymes, was not detected in the klf17-deficient embryos (Fig. 5). Furthermore, the expression of polster and hatching gland marker genes, he1.1, ctsl1b and $c d 63$ was reduced in the klf17-deficient embryos at the bud stage and $25 \mathrm{hpf}$ (Figs 6 and 7). The polster at the bud stage was not detected in the klf17-deficient embryo. Therefore, the klf17 gene is an indispensable transcription factor for the polster and hatching gland development in zebrafish. If the 

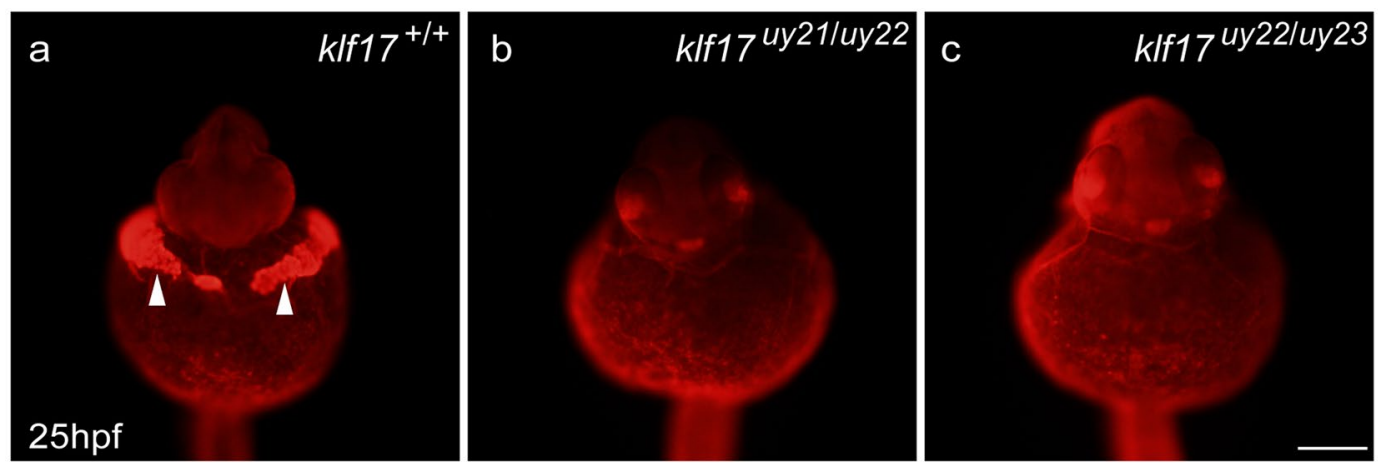

Figure 5. Cathepsin $1 \mathrm{lb}$ protein expression in the hatching gland. The expression of Cathepsin L $1 \mathrm{~b}$ (Ctsl1b), which is one of hatching gland enzymes, was examined using whole-mount immunohistochemistry at 25 hpf. (a) Wild-type $\left(k l f 17^{+/+}\right),(\mathbf{b}, \mathbf{c})$ klf17-deficient embryos: $k l f 17^{\text {uy21/uy22 }}$ (b) and $k l f 17^{\text {uy22/uy23 }}$. (c) Ventral view, anterior up. Arrowheads indicate the position of the hatching gland. After taking pictures, genotyping of individual embryos was performed by genomic PCR. Scale bar, $200 \mu \mathrm{m}$.

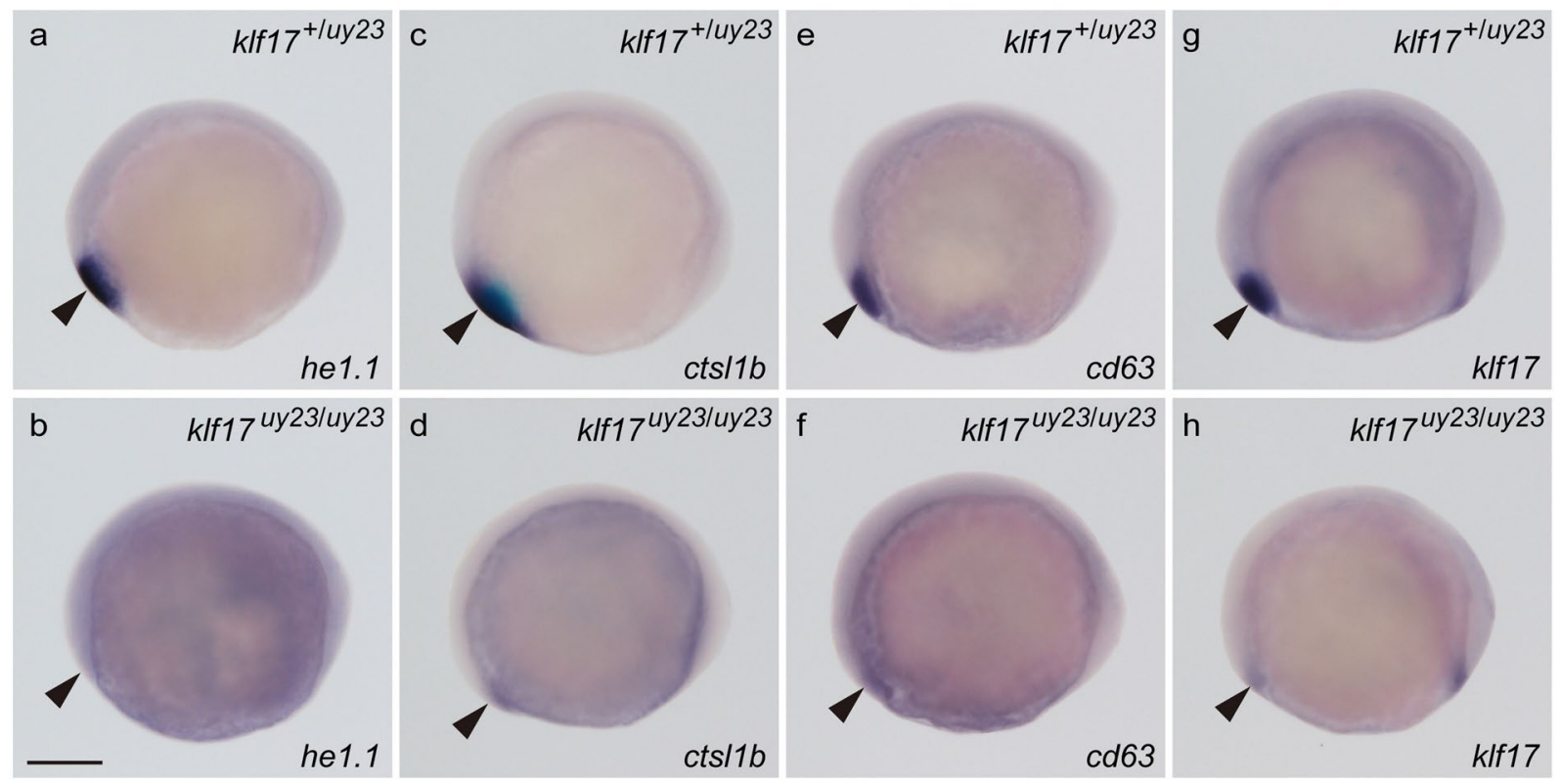

Figure 6. The expression of polster genes in the klf17-deficient embryos at the bud stage. The expression of polster markers he1.1 (a,b), ctsl1b (c,d), $c d 63(\mathbf{e}, \mathbf{f})$ and $k l f 17(\mathbf{g}, \mathbf{h})$ was examined by WISH at the bud stage. $(\mathbf{a}, \mathbf{c}, \mathbf{e}, \mathbf{g})$ Wild-type embryos $\left(k l f 17^{+/ u y 23}\right)$. (b,d,f,h) klf17-deficient embryos $\left(k l f 17^{\text {uy23/uy23 }}\right)$. Lateral view, anterior left. Arrowheads indicate the position of the polster. After taking pictures, genotyping of individual embryos was performed by genomic PCR. Scale bar, $200 \mu \mathrm{m}$.

physiological function of the $k l f 17$ gene is conserved among fish, the disruption of fish $k l f 17$ genes mediated by genome editing technologies may be useful for eliminating invasive alien fish in closed areas.

\section{Methods}

Ethics statement. All animal experiments were performed in accordance with the animal protocol approved by the Institutional Animal Care and Use Committee (IACUC) of the University of Yamanashi. The IACUC of the University of Yamanashi approved this study (Approval Identification Number: A25-28).

Synthetic crRNA and tracrRNA, recombinant Cas9 protein and microinjection. To disrupt the targeted klf17 genomic locus, we used the ready-to-use CRISPR/Cas9 system composed of CRISPR RNA (crRNA), trans-activating crRNA (tracrRNA) and recombinant Cas 9 protein ${ }^{23}$. Synthetic crRNAs and tracrRNA (Supplementary Table S1), and recombinant Cas9 protein were obtained from the Integrated Device Technology, Inc (IDT). Synthetic klf17-crRNA1 (25 pg), klf17-crRNA2 (25 pg) and tracrRNA (100 pg) were co-injected together with recombinant Cas9 protein (1 ng) into 1-cell stage zebrafish embryos. Klf17-morpholinos (klf17-MO, 
a

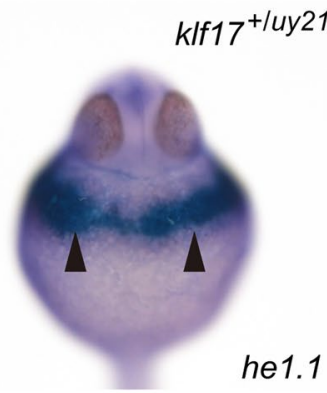

b

he1.1
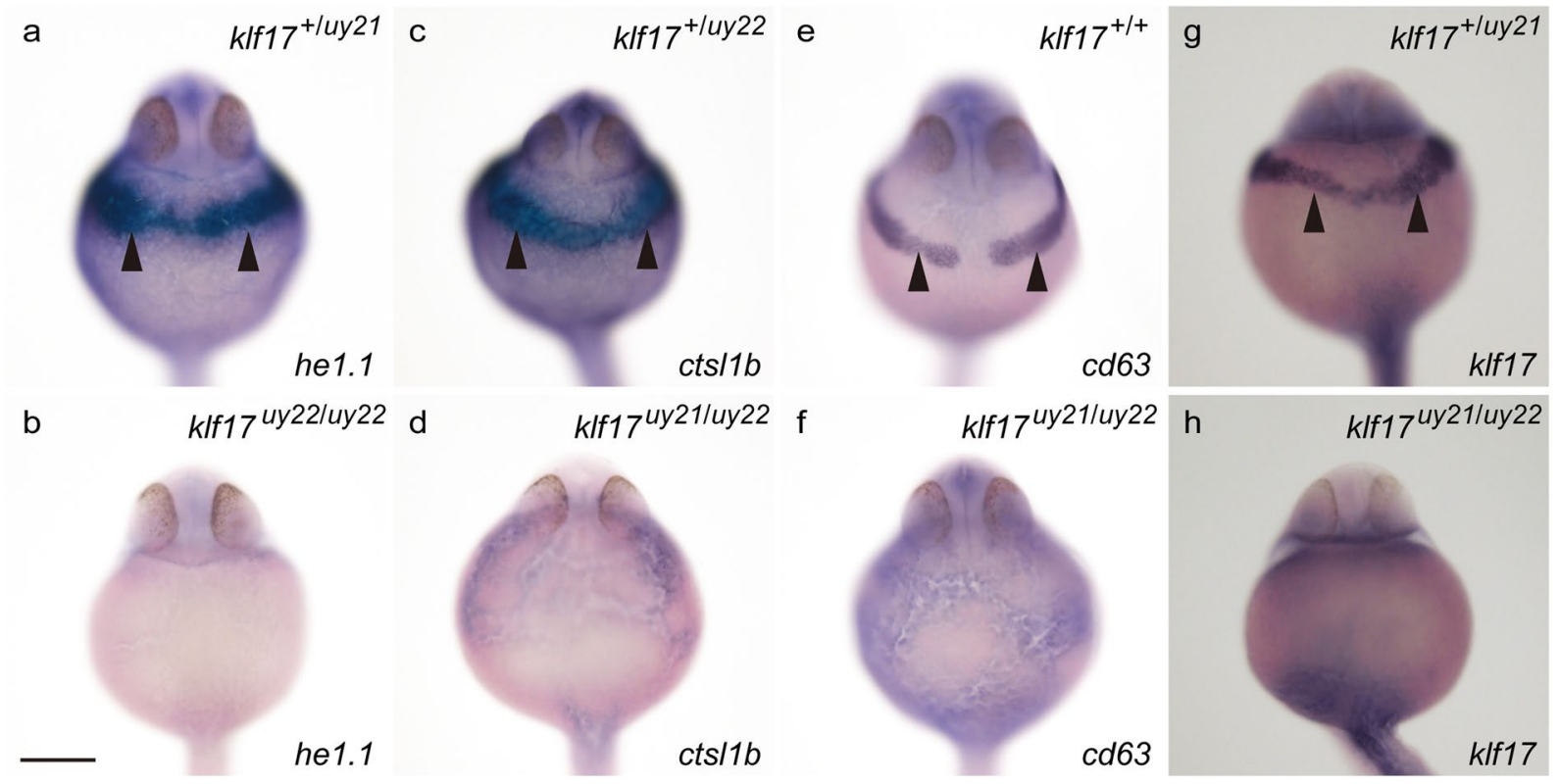

Figure 7. The expression of hatching gland genes in the klf17-deficient embryos at $25 \mathrm{hpf}$ (a,c,e,g) Wild-type embryos at 25 hpf. (b,d,f,h) klf17-deficient embryos at $25 \mathrm{hpf}$. Ventral view, anterior up. Arrowheads indicate the position of the hatching gland. After taking pictures, genotyping of individual embryos was performed by genomic PCR. Scale bar, $200 \mu \mathrm{m}$.

5'-TGCAAATGTTAGGGAACTCAGAAGG-3') were injected at one-cell stage embryos as described previously $^{24}$. klf17 mRNA (20 pg) was injected into blastomere at one-cell stage embryos.

Genotyping for the $\boldsymbol{k l f} \mathbf{1 7}$ locus and genomic sequencing. To prepare the genomic DNA, the embryos at indicated stages were incubated in $108 \mu \mathrm{l}$ of $50 \mathrm{mM} \mathrm{NaOH}$ at $98^{\circ} \mathrm{C}$ for $10 \mathrm{~min}$. Subsequently, $12 \mu \mathrm{l}$ of $1 \mathrm{M}$ Tris- $\mathrm{HCl}$ ( $\mathrm{pH} 8.0$ ) was added to the solution ${ }^{23}$. Genomic fragments at the targeted sites were amplified by PCR with PrimeTaq (GENETBIO Inc.) and the locus-specific primers are listed in Supplementary Table S2. The PCR conditions were as follow: 40 cycles of $98^{\circ} \mathrm{C}$ for $10 \mathrm{~s}, 55^{\circ} \mathrm{C}$ for $30 \mathrm{~s}$ and $72^{\circ} \mathrm{C}$ for $30 \mathrm{~s}$. To perform heteroduplex mobility assay (HMA) for genotyping, the resultant PCR amplicons were electrophoresed on a $12.5 \%$ polyacrylamide gel ${ }^{25}$. To confirm individual mutations, genomic fragments for the targeted genomic locus were amplified from the solution $(1 \mu \mathrm{l})$ using PCR (Supplementary Table S2). The resultant PCR fragments were sub-cloned into the pGEM-T Easy vector (Promega) and genomic sequences were determined by sequence analysis.

Lateral line neuromast labeling. Lateral line neuromasts at the $54 \mathrm{hpf}$ stage embryos were labeled by incubation of live fish with $0.5 \mathrm{mM} 4$-(4-diethylaminostyryl)- $\mathrm{N}$-methylpyridinium iodide (4-Di-2-ASP) in E3 $\left(5 \mathrm{mM} \mathrm{NaCl}, 0.17 \mathrm{mM} \mathrm{KCl}, 0.33 \mathrm{mM} \mathrm{CaCl}_{2}\right.$ and $0.33 \mathrm{mM} \mathrm{MgSO}_{4}$ ) medium for $7 \mathrm{~min}$. Labelled fish were washed 3 times with $\mathrm{E} 3$ medium and anaesthetized with tricaine (3-amino benzoic acidethylester), and subsequently were observed under fluorescence microscope. For lateral line neuromast labeling with alkaline phosphatase ${ }^{26}$, the embryos at $54 \mathrm{hpf}$ were fixed in $4 \%$ paraformaldehyde for $3 \mathrm{~h}$ at room temperature and washed with phosphate buffered saline plus $0.1 \%$ Tween-20 (PBST). The embryos were developed in alkaline phosphate buffer containing NBT and BCIP (Nacalai tesque) for $30 \mathrm{~min}$.

Histological analysis. Embryos were dehydrated in a graded series of ethanol and embedded using a Technovit 8100 kit (Kulzer). Embedded embryos were sectioned on a Leica RM2125 microtome at $6 \mu \mathrm{m}$ and mounted on slides. Embryos were stained with haematoxylin-eosin (HE) after sectioning.

Whole-mount immunohistochemistry. Embryos were incubated with anti-Cathepsin L 1b (GeneTex, Inc.) at $4{ }^{\circ} \mathrm{C}$ overnight in PBST containing 5\% sheep serum and washed 4 times with PBST. Subsequently, embryos were incubated with Alexa Fluor 594 goat anti-rabbit IgG (Invitrogen) at room temperature for $4 \mathrm{~h}$ in PBST containing 5\% sheep serum and washed 4 times with PBST.

Whole-mount in situ hybridization (WISH). We examined the expression of $s 100 t^{27}$, he1.1, ctsl1 $b^{28}$, $c d 63^{29}, k l f 17$, gata 1, $\beta_{\mathrm{e} 3}$ globin and lyz. Whole-mount in situ hybridization (WISH) was performed as previously described $^{30}$. Zebrafish embryos hybridized with the digoxigenin (DIG)-labelled RNA probe were incubated with alkaline phosphatase-conjugated anti-DIG antibody. To visualize the RNA probe recognized by the anti-DIG antibody, the embryos were subsequently incubated with BM Purple (Roche) as the substrate. Washing the embryos with PBST terminated the colour reaction, and the embryos were fixed in $4 \%$ paraformaldehyde. 


\section{References}

1. Kaczynski, J., Cook, T. \& Urrutia, R. Sp1- and Krüppel-like transcription factors. Genome Biol. 4, 206 (2003).

2. McConnell, B. B. \& Yang, V. W. Mammalian Krüppel-like factors in health and diseases. Physiol. Rev. 90, 1337-1381 (2010).

3. Kotkamp, K., Mössner, R., Allen, A., Onichtchouk, D. \& Driever, W. A Pou5f1/Oct4 dependent Klf2a, Klf2b, and Klf17 regulatory sub-network contributes to EVL and ectoderm development during zebrafish embryogenesis. Dev. Biol. 385, 433-447 (2014).

4. Yan, W., Burns, K. H., Ma, L. \& Matzuk, M. M. Identification of Zfp393, a germ cell-specific gene encoding a novel zinc finger protein. Mech. Dev. 118, 233-239 (2002).

5. Zhou, S., Tang, X. \& Tang, F. Krüppel-like factor 17, a novel tumor suppressor: its low expression is involved in cancer metastasis. Tumor Biol. 37, 1505-1513 (2016).

6. Gumireddy, K. et al. KLF17 is a negative regulator of epithelial-mesenchymal transition and metastasis in breast cancer. Nat. Cell Biol. 11, 1297-1304 (2009).

7. Kawahara, A. \& Dawid, I. B. Expression of the Krüppel-like zinc finger gene biklf during zebrafish development. Mech. Dev. 97, 173-176 (2000).

8. Huber, T. L. et al. Naptune, a Krüppel-like transcription factor that participates in primitive erythropoiesis in Xenopus. Curr. Biol. 11, 1456-1461 (2001)

9. Takeda, M., Kurauchi, T., Yamazaki, T., Izutsuh, Y. \& Maéno, M. Naptune is involved in posterior axis and tail formation in Xenopus embryogenesis. Dev. Dyn. 234, 63-73 (2005).

10. Antin, P. B., Pier, M., Sesepasara, T., Yatskievych, T. A. \& Darnell, D. K. Embryonic expression of the chicken krüppel-like (KLF) transcription factor gene family. Dev. Dyn. 239, 1879-1887 (2010).

11. Kawahara, A. \& Dawid, I. B. Critical role of biklf in erythroid cell differentiation in zebrafish. Curr. Biol. 11, 1353-1357 (2001).

12. Gardiner, M. R., Daggett, D. F., Zon, L. I. \& Perkins, A. C. Zebrafish KLF4 is essential for anterior mesendoderm/pre-polster differentiation and hatching. Dev. Dyn. 234, 992-996 (2005).

13. Gardiner, M. R., Gongora, M. M., Grimmond, S. M. \& Perkins, A. C. A global role for zebrafish klf4 in embryonic erythropoiesis. Mec. Dev. 124, 762-774 (2007).

14. Kurauchi, T., Izutsu, Y. \& Maéno, M. Invovement of Naptune in induction of the hatching gland and neural crest in the Xenopus embryo. Differentiation 79, 251-259 (2010).

15. Kok, F. O. et al. Reverse genetic screening reveals poor correlation between morpholino-induced and mutant phenotypes in zebrafish. Dev. Cell 32, 97-108 (2015).

16. Robu, M. E. et al. p53 activation by knockdown technologies. PLoS Genet. 3, e78 (2007).

17. Gerety, S. S. \& Wikinson, D. G. Morpholino artifacts provide pitfalls and reveal a novel role for pro-apoptotic genes in hindbrain boundary development. Dev. Biol. 350, 279-289 (2011).

18. Aman, A., Nguyen, M. \& Piotrowski, T. Wnt/ $\beta$-catenin dependent cell prolifereation underlies segmented lateral line morphogenesis. Dev. Biol. 349, 470-482 (2011).

19. Sapède, D., Gompel, N., Dambly-Chaudrière, C. \& Ghysen, A. Cell migration in the postembryonic development of the fish lateral line. Development 129, 605-615 (2002).

20. Xue, Y., Gao, S. \& Liu, F. Genome-wide analysis of the zebrafish Klf family identifies two genes important for erythroid maturation. Dev. Biol. 403, 115-127 (2015).

21. Ghysen, A. \& Dambly-Chaudrière, C. The lateral line microcosmos. Genes Dev. 21, 2118-2130 (2007).

22. De la Paz, J. F., Beiza, N., Paredes-Zúñiga, S., Hoare, M. S. \& Allende, M. L. Triazole fungicides inhibit zebrafish hatching by blocking the secretory function of hatching gland cells. Int. J. Mol Sci. 18, E710 (2017).

23. Kotani, H., Taimatsu, K., Ohga, R., Ota, S. \& Kawahara, A. Efficient multiple genome modifications induced by the crRNAs, tracrRNA and Cas9 Protein complex in zebrafish. PLoS One 10, e0128319 (2015).

24. Kitaguchi, T., Kawakami, K. \& Kawahara, A. Transcriptional regulation of a myeloid-lineage specific gene lysozyme C during zebrafish myelopoiesis. Mech. Dev. 126, 314-323 (2009).

25. Ota, S., Hisano, Y., Ikawa, Y. \& Kawahara, A. Multiple genome modifications by the CRISPR/Cas 9 system in zebrafish. Genes Cells 19, 555-564 (2014).

26. Chitramuthu, B. P. et al. Molecular cloning and embryonic expression of zebrafish pcsk 5 co-orthologues: functional assessment during lateral line development. Dev. Dyn. 239, 2933-2946 (2010).

27. Venero Galanternik, M., Kramer, K. L. \& Piotrowski, T. Heparin sulfate proteoglycans regulate fgf signaling and cell polarity during collective cell migration. Cell Rep. 10, 414-428 (2015).

28. Vogel, A. M. \& Gerster, T. Expression of a zebrafish Cathepsin L gene in anterior mesendoderm and hatching gland. Dev. Genes Evol. 206, 477-479 (1997).

29. Trikić, M. Z., Monk, P., Roehl, H. \& Partidge, L. J. Regulation of zebrafish hatching by tetraspanin cd63. PLoS One 6, e19683 (2011).

30. Kawahara, A. et al. The sphingolipid transporter Spns2 functions in migration of zebrafish myocardial precursors. Science 323, 524-527 (2009).

\section{Acknowledgements}

We thank A. Nagase for zebrafish maintenance, T. Mori, T. Miyagi, H. Morita and Y. Sugano for technical assistance and advice and T. Saigoh and M. Itoh for helpful discussion. This work was supported by grants from the Ministry of Education, Science, Technology, Sports and Culture of Japan and the Takeda Science Foundation, Japan.

\section{Author Contributions}

A.K. conceived and designed the work and wrote the manuscript. H.S., T.I., K.Y., R.S., Y.S., R.O. and A.K. performed the experiments. All authors performed the data analysis and reviewed the manuscript.

\section{Additional Information}

Supplementary information accompanies this paper at https://doi.org/10.1038/s41598-019-50149-5.

Competing Interests: The authors declare no competing interests.

Publisher's note Springer Nature remains neutral with regard to jurisdictional claims in published maps and institutional affiliations. 
(c) (i) Open Access This article is licensed under a Creative Commons Attribution 4.0 International License, which permits use, sharing, adaptation, distribution and reproduction in any medium or format, as long as you give appropriate credit to the original author(s) and the source, provide a link to the Creative Commons license, and indicate if changes were made. The images or other third party material in this article are included in the article's Creative Commons license, unless indicated otherwise in a credit line to the material. If material is not included in the article's Creative Commons license and your intended use is not permitted by statutory regulation or exceeds the permitted use, you will need to obtain permission directly from the copyright holder. To view a copy of this license, visit http://creativecommons.org/licenses/by/4.0/.

(C) The Author(s) 2019 